\title{
Low energy indoor network: deployment optimisation
}

\author{
Siyi Wang*, Weisi Guo and Tim O'Farrell
}

\begin{abstract}
This article considers what the minimum energy indoor access point deployment is in order to achieve a certain downlink quality-of-service. The article investigates two conventional multiple-access technologies, namely: LTEfemtocells and 802.11n Wi-Fi. This is done in a dynamic multi-user and multi-cell interference network. Our baseline results are reinforced by novel theoretical expressions. Furthermore, the work underlines the importance of considering optimisation when accounting for the capacity saturation of realistic modulation and coding schemes. The results in this article show that optimising the location of access points both within a building and within the individual rooms is critical to minimise the energy consumption.
\end{abstract}

\section{Introduction}

Recent research shows that more than $50 \%$ of voice calls and more than $70 \%$ of data traffic are generated indoors [1]. Two main wireless technologies have been used for serving users indoors. The first one is the traditional outdoor cellular system which deals with real time voice, short messages and mobile broadband (MBB) applications. The other is wireless local area networks (WLANs) which focus on providing non-real time data applications [2]. Due to the increasing demands for indoor higher data-rate wireless applications, existing cellular systems will be insufficient to meet the expected quality of service (QoS) requirement for indoor users from both service coverage and capacity perspectives. The femtocell access points (FAPs) have been proposed to address the above challenge, which uses low-power, short-range and low-cost base stations. Femtocells are compatible with the existing outdoor macro-cell cellular base stations which can easily support seamless handoff but provide better indoor signal strength. With the introduction of femtocell technology, serving base stations are becoming similar to the closest competing Wi-Fi technology in reality. Instead of the conventional cellular network infrastructure, femtocells use the IP Network as a backhaul architecture which has a lot in common with the existing 802.11 technology. Despite the huge potentials of femtocells, they still face many technical and business challenges. Whilst existing research has

\footnotetext{
* Correspondence: siyi.wang@sheffield.ac.uk

Department of Electronic and Electrical Engineering, The University of Sheffield, Mappin Street, Sheffield, S1 3JD, UK
}

focused on dynamic power control and radio resource management to mitigate interference between indoor and the outdoor network, the issue of the physical deployment of the indoor network (the number and location of indoor access points (APs)) is unresolved.

\subsection{Review of existing work}

Several existing research [3-5] has been focusing on the improvement of femtocell network throughput. Al-Rubaye et al. [3] outlined the cognitive radio technologies for the future $\mathrm{MBB}$ era by proposing a cognitive femtocell solution for indoor communications in order to increase the network capacity in serving indoor users and to solve the spectrum-scarcity problems. Ko and Wei [4] proposed a desirable resource allocation mechanism, taking into account mobile users' selfish characteristics and private traffic information to improve the femtocell network performance. The aggregate throughput of two-tier femtocell networks has been improved by a beamforming codebook restriction strategy and an opportunistic channel selection strategy in [5]. However, the above study did not take into account the location and the number of FAPs deployed in the indoor environment.

There also have been various approaches to investigate the optimal base station (BS) placement to achieve the operator's desired QoS or coverage targets. Much of the previous work [6-8] has focused on minimising the transmitting power of BS. Fagen et al. [9] proposed a new automated method of simultaneously maximising coverage while minimising interference for a desired level of (c) 2012 Wang et al; licensee Springer. This is an Open Access article distributed under the terms of the Creative Commons Attribution License (http://creativecommons.org/licenses/by/2.0), which permits unrestricted use, distribution, and reproduction in any medium, provided the original work is properly cited. 
coverage overlap. However, such an approach is not always practical as network optimisation is constrained by a number of restrictions on BS placements, interference and power emissions. Ashraf et al. [10] described an approach of adjusting the transmitting power of fixed positions of FAPs in the enterprise offices to achieve coverage optimisation and load balance, but did not consider the evaluation of the effect on the positions of FAPs. In [11], a joint power and channel allocation method was proposed to improve the uplink throughput, but the calculation of the throughput, which was the key parameter of the algorithm, was relatively simple and might be inaccuracy by just using Shannon capacity equation. Ki Wo et al. [12] derived the downlink SINR formula for the residential femtocell but the formula did not take the throughput into account while researchers in [13] provided system simulation to evaluate the femtocell network performance, however the simulation was relatively simple without an extensive theoretical model verification. A theoretical framework was proposed in [14] to analyse the interference characteristics of different femtocell sub-bands for Orthogonal Frequency-Division Multiple Access (OFDMA) systems employing the Fractional Frequency Reuse scheme which could be extended to optimise power and frequency allocation, but the pathloss model employed in this framework is far too simple to reflect the real characteristics of the indoor scenario.

Bianchi proposes a classic two-dimensional Markov chain to determine the saturation throughput of a Wireless Local Area Network (WLAN) using the Distributed Coordination Function (DCF) [15]. Tay and Chua [16] proposed a model based on average value analysis and studied the effects of contention window sizes on the throughput performance. Both of the above models assumed an ideal wireless channel with no physical layer (PHY) channel errors. In fact, wireless channels are usually error-prone and the packet errors have an impact on the system performance. Several articles has extended the above system models to study the throughput performance under different channel error conditions.

\subsection{Contribution}

Given the large number of propagation variables in indoor buildings and its relation to the outdoor cellular network, the article provides a best practice in optimising AP deployment with very little signal to interferenceplus-noise ratio (SINR) degradation for micro-cell users. The novel contribution is the simulation results and the theoretical framework that reinforces the key deployment solutions. Our baseline results are reinforced by novel theoretical expressions. Moreover, for a given building size, the trade-off between increased QoS and power consumption, as well as the capacity saturation points are demonstrated. An approach has been introduced to study the saturated throughput, user QoS and energy consumption performances of $802.11 \mathrm{n}$ networks under error-prone channels by extending Bianchi's model.

\section{Body of investigation}

The article considers two indoor deployment scenarios and shows that there is a shared principle of deployment between them:

- Concept demonstration: Single Room with and without an outdoor interference source (Simulation and theory);

- Generic building with multiple rooms on multiple floors with an outdoor interference source (Simulation).

The combined results of the two scenarios will lead to a general low energy indoor deployment rule. Moreover, the single room femtocell results are reinforced with a novel theoretical framework that can optimise the location of an AP with respect to the interference and propagation parameters. It is shown that the key results hold for a multiple room building. There is only 1 outdoor interference source considered, because given that a building is inside the coverage of a cell, the interference of that cell will be far greater than neighbouring cells that are further away.

In the baseline conventional scenario (Figure 1a), 802.11 $\mathrm{n}$ APs are deployed on three non-overlapping channels using a total bandwidth of $60 \mathrm{MHz}$ while FAPs are assumed to operate on the same frequency with a total bandwidth of $20 \mathrm{MHz}$. In the alternative scenario (Figure 1b), both $802.11 \mathrm{n}$ APs and FAPs have a total bandwidth of $20 \mathrm{MHz}$ with different frequency reuse pattern 1 and 3, respectively. The system model and theoretical framework for FAP considers the following setup as shown in Figures 1c,d.

The article aims to provide the comparison between these two technologies in their basic service in reality, hence the advanced feature of $802.11 \mathrm{n}$ such as frame aggregation to MAC layer and multiple-input multipleoutput (MIMO) and $40 \mathrm{MHz}$ channels to the PHY are outside the scope of this study.

- LTE-femtocell Simulation: Co-channel FAPs employing SISO transmission and Round Robin (RR) scheduling. The link level capacity is derived from adaptive modulation and coding schemes. This is simulated for a single room and a building with multiple rooms.

- 802.11n Wi-Fi Simulation: Wi-Fi APs employing frequency reuse 1 and 3 , and SISO transmission 


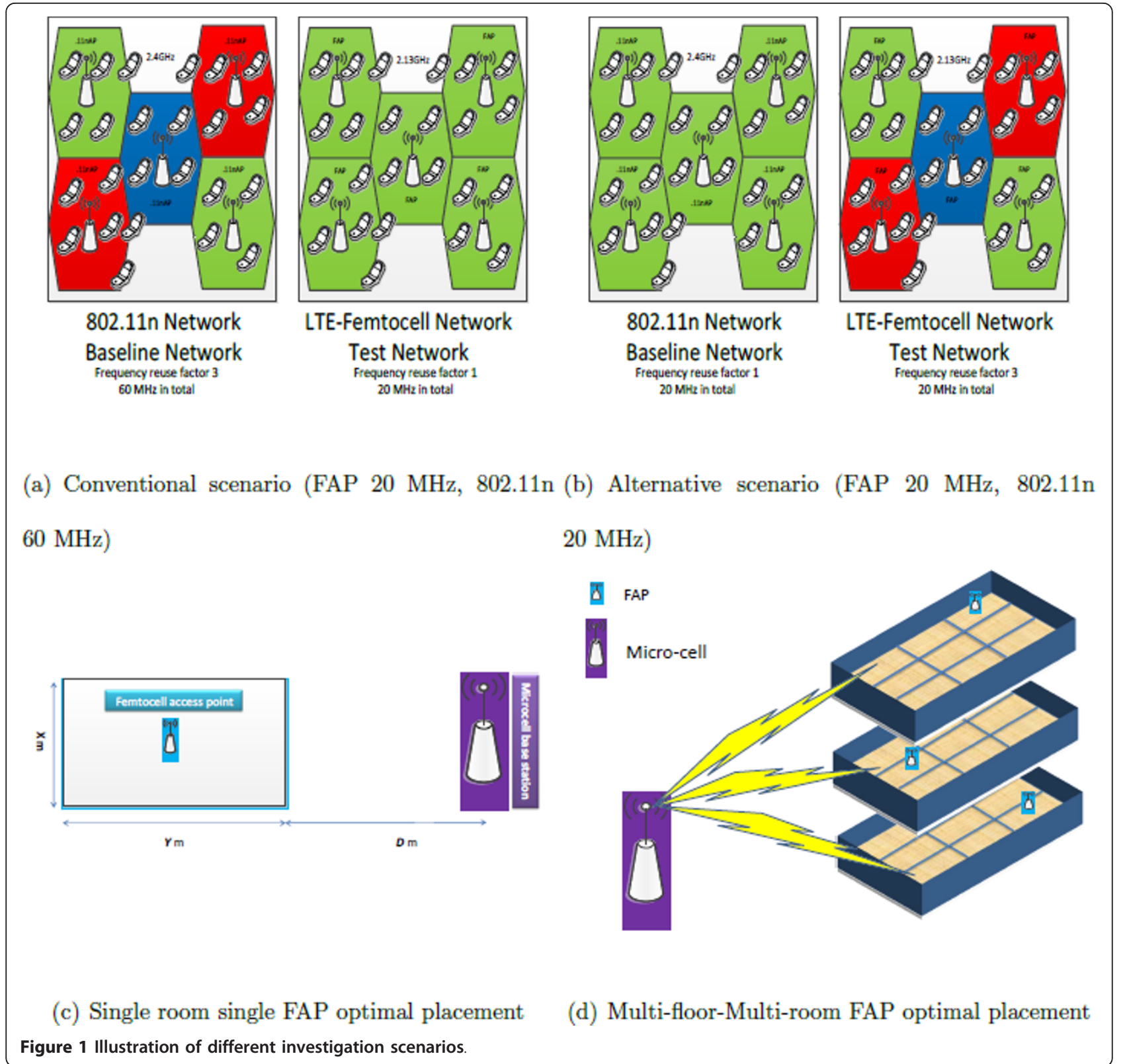

with a theoretical contention model. The link level capacity is derived from adaptive modulation and coding schemes. This is simulated for a single room.

- Single Room Theory: Single LTE-femtocell deployed in a single room in the presence of a fully loaded outdoor micro-cell BS.

\subsection{LTE-femtocells simulator model}

The user distribution for each room or building is random even distribution. The position of each user and the traffic conditions are updated within each loop and the simulation results are run enough times to reach convergence. RR scheduler is employed, which evenly partitions the resource blocks between users. In this simulator resource allocation is performed at intervals of $1 \mathrm{~ms}$ in the time domain. This interval is called a transmission time interval (TTI) [17].

The path loss models implemented in the simulator were adopted from WINNER II [18]. The indoor path loss model $\mathrm{PL}_{\text {in }}$ in $\mathrm{dB}$ (between the FAP and the mobile user) and the outdoor-to-indoor $\mathrm{PL}_{\text {out-to-in }}$ in $\mathrm{dB}$ (between the micro-cell BS and the mobile user) are defined as follows, respectively:

$$
\mathrm{PL}_{\mathrm{in}}=18.7 \log _{10}\left(d_{\mathrm{FAP}}\right)+46.8+20 \log _{10}\left(\frac{f}{5}\right)
$$




$$
\mathrm{PL}_{\text {out-to-in }}=36.7 \log _{10}\left(d_{\text {micro }}\right)+22.7+26 \log _{10}\left(\frac{f}{5}\right)+\mathrm{PL}_{\text {wal1 } 1}+0.5 d_{\text {in }}
$$

where $f$ is frequency of transmission in GHz. $d_{\mathrm{FAP}}$, $d_{\text {micro }}$ and $d_{\text {in }}$ are FAP-to-user, micro-cell-to-user and wall-to-user distance in metres. $\mathrm{PL}_{\text {wall }}$ is the wall loss penetration factor in $\mathrm{dB}$. The received SINR is calculated as below:

$$
\gamma=\frac{\left|h_{i}\right|^{2} P_{i}}{\sum_{k=1, k \neq 1}^{K}\left|h_{k}\right|^{2} P_{k}+\left|h_{\text {micro }}\right|^{2} P_{\text {micro }}+\sigma^{2}},
$$

where $h_{i}, h_{k}$ and $h_{\text {micro }} \sim \mathcal{C N}(0,1)$ are the multi-path coefficient of the observed FAP, interfering FAPs and micro-cell BS, respectively. They are modelled as independent and identically distributed (i.i.d.) circularly symmetric complex Gaussian random variables with zero mean and a variance of one. $P_{i}$ is the received power of one sub-carrier from the observed FAP, $P_{k}$ and $P_{\text {micro }}$ are the received power of the same sub-carrier from the interfering FAPs and micro-cell BS and $\sigma^{2}$ is the noise power. The simulator supports link adaptation by changing the Modulation and Coding Scheme (MCS) based on the channel quality $\gamma$ (i.e. SINR). The MCS look-up tables was generated from the Vienna link level LTE simulator [19] under WINNER II indoor multipath model [18]. The corresponding block error rate (BLER) and throughput versus signal to noise ratio (SNR) curves of the look-up tables are illustrated in Figure 2. The instantaneous user data rate in each TTI is calculated by the multiplication of the number of bits per resource element obtained from the relative link adaptation table and the number of resource elements that a user has been assigned. The RAN capacity is then calculated on the basis of each user's data rate. User QoS requirement is defined as a threshold on the percentage of users that can achieve the target data rate. From the network service point of view, a technology with a specific network configuration can be said to satisfy the network QoS requirement only if the percentage of users that achieve the targeted data rate is larger than the percentage threshold of User QoS, under any given network topology. For example, the QoS requirements for the network service is set to $0.5 \mathrm{Mbit} / \mathrm{s}$ target data rate and 95\%-ile threshold in this report, it means that the network satisfies QoS requirements only if at least 95\% of users achieve a minimum data rate of $0.5 \mathrm{Mbit} / \mathrm{s}$. The RAN QoS is computed by multiplying the user QoS and the number of the users in that RAN.

\section{$2.2802 .11 \mathrm{n}$ simulator}

The $802.11 \mathrm{n}$ simulator is based on an existing throughput analytical model [15]. The model is concerned with

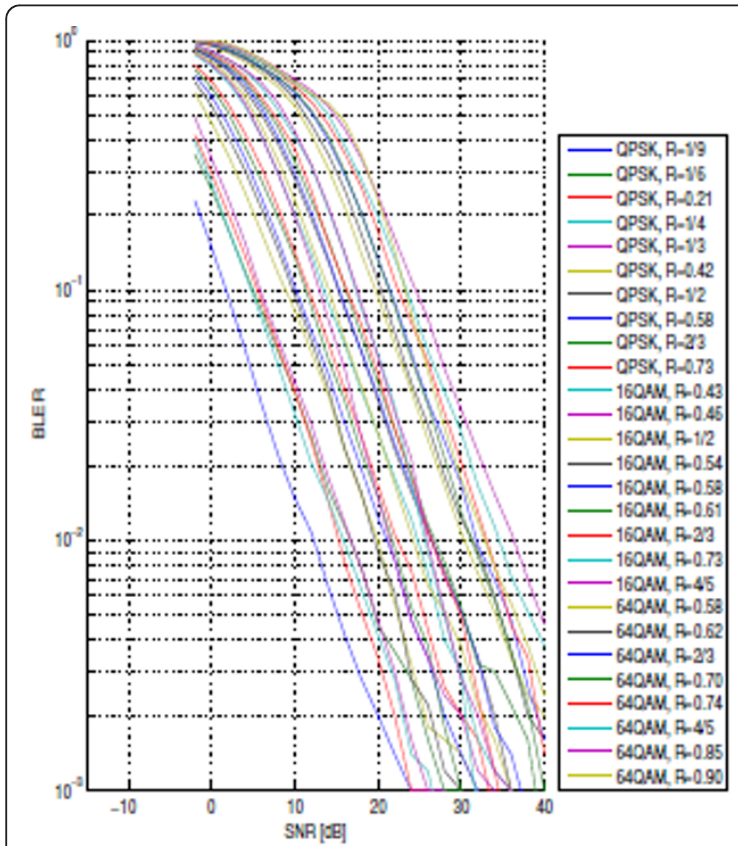

(a) BLER vs. SNR WINNER_II A1 SISO

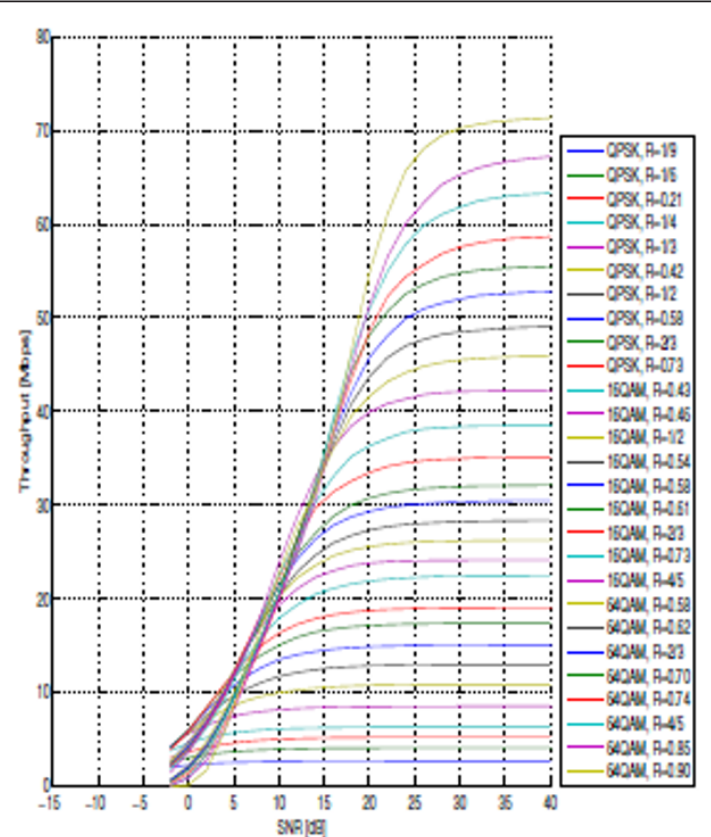

(b) Throughput vs. SNR WINNER_II A1SISO

Figure 2 BLER and throughput versus SNR plots for the 27 MCSs with SISO antenna configurations. 
infrastructure mode WLANs that use the DCF medium access control (MAC) protocol. The model assumes that there are a number of $802.11 \mathrm{n}$ APs operating on three different frequency channels in conventional scenario and one frequency channel in deployed indoors and a fixed number of client stations in the WLAN. Each user is associated with exactly one AP which provides the highest SINR to that user and each AP with its associated stations defines a cell. Therefore, DCF is used for single-hop only communication within the cells and users access data through their serving APs. Each user is assumed to have saturated traffic. The wireless channel bit error rate (BER) is $P_{b}$. The minimum contention window size is $W$ and the maximum backoff stage is $m$. In 802.11 WLANs, control frames are transmitted at the basic rate which is more robust in combating errors. They have a much lower frame error rate as the size of these control frames are much smaller than an aggregated data frame. Therefore, the frame error probabilities for control frames and preambles are assumed to be zero.

The system time is divided into small time slots where each slot is the time interval between two consecutive countdowns of backoff timers by stations which are not transmitting. From Bianchi's model, transmission probability $\tau$ in a virtual time slot is given by:

$$
\tau=\frac{2(1-2 p)}{(1-2 p)(W+1)+p W(1-2 p)^{m}},
$$

where $p$ is the unsuccessful transmission probability conditioned on that there is a transmission in a time slot. When considering both collisions and errors, $p$ can be expressed as:

$$
p=1-\left(1-p_{c}\right)\left(1-p_{e}\right),
$$

where $p_{c}=1-(1-\tau)^{n-1}$ is the packet conditional collision probability and $p_{e}=1-\left(1-p_{b}\right)^{L}$ is the packet error probability on condition that there is a successful transmission in the time slot. $n$ is the total number of contending stations. $L$ is the packet size in bits and $p_{b}$ is the BER of a particular MCS level. Therefore, the network saturation throughput can be calculated as: $S=\frac{E_{p}}{E_{t}}$, where $E_{p}$ is the average packet payload bits successfully transmitted in a virtual time slot, and $E_{t}$ is the expected length of a time slot. $E_{p}$ and $E_{t}$ are computed by (6) and (7):

$$
\begin{aligned}
& E_{p}=L p_{s}=L p_{t r} p_{s_{-} n c}\left(1-p_{e}\right), \\
& E_{t}=T_{\sigma} p_{\sigma}+T_{c} p_{t r}\left(1-p_{s_{-} n c}\right)+T_{e} p_{e}+T_{s} p_{s},
\end{aligned}
$$

where the probability of an idle slot $p_{\sigma}$ is $(1-\tau)^{n}$, the probability of a non-collided transmission $p_{s_{-} n c}$ is $\frac{n(1-\tau)^{n-1}}{p_{t r}}$, the probability for a transmission in a time slot $p_{t r}$ is $1-p_{\sigma}=1-(1-\tau)^{n}$, the probability of a successful transmission (without collisions and transmission errors) is $p_{t r} p_{s_{n} n c}\left(1-p_{e}\right)$ and $\tau$ is computed by (4). $T_{\sigma}$ is equal to the system's empty slot time of $9 \mu \mathrm{s} . T_{\sigma}, T_{c} T_{s}$ and $T_{e}$ are the idle, collision, successful and error virtual time slot's length and are defined as follows: $T_{c}=$ EIFS, $T_{s}=\mathrm{DATA}+\mathrm{BACK}+3 \mathrm{SIFS}+\mathrm{DIFS}, T_{e}=\mathrm{DATA}+$ EIFS +2 SIFS, where BACK $=5.63 \mu$ s and DATA are the transmission time for backoff stage and the transmission time for aggregated data frame, SIFS $=16 \mu \mathrm{s}$, DIFS $=$ SIFS $+T_{\sigma}$, EIFS $=$ SIFS + DIFS + BACK, respectively. 48 of the 52 OFDM sub-carriers are for data and the remaining 4 are for pilot sub-carriers.

\section{Power and energy metrics}

The power consumption model employed by this article considers the FAP and 802.11n APs to have the same model. This assumption is reinforced by existing literature [20]. The model considers the power consumption of an AP to have two distinct parts: a radio-head (RH) and an overhead $(\mathrm{OH})$. Together the $\mathrm{RH}$ and $\mathrm{OH}$ constitute the operational (OP) power consumption of the AP. During transmission, the RH is active, and irrespective of transmission, the fixed $\mathrm{OH}$ is always active.

In order to compare the energy consumption of the same system operating in different conditions, the concept of transmission and OP duration are defined. Consider an AP with indoor users that demand a traffic load of $M$ bits of data over a finite time duration of $T_{\mathrm{AP}}^{\mathrm{OH}}$. Two systems are considered: a reference and a test system, both of which have a capacity that exceed the offered traffic load. Due to the fact that the reference and the test system might have different throughput, the duration which the $\mathrm{RH}$ spends in transmitting the same $M$ bits is different. In order to compare the energy of the two systems, a useful metric is the energy reduction gain (ERG), which is the reduction in energy consumption when a test system is compared with a reference system:

$$
\mathrm{ERG}_{\mathrm{RAN}}^{\mathrm{OP}}=1-\frac{E_{\mathrm{AP}, \text { test }}^{\mathrm{OH}}}{E_{\mathrm{AP}, \text { ref. }}^{\mathrm{OP}}}=1-\frac{n P_{\text {test }}^{\mathrm{RH}} \frac{R_{\text {tafficic }}}{R_{\mathrm{AP} \text { test }}}+n P_{\text {test }}^{\mathrm{OH}}}{n P_{\text {ref. }}^{\mathrm{RH}} \frac{R_{\text {traffic }}}{R_{\mathrm{AP}, \text { ref. }}}+n P_{\text {ref. }}^{\mathrm{OH}}},
$$

where $P_{i}^{\mathrm{RF}}, P_{i}^{\mathrm{RH}}$ and $P_{i}^{\mathrm{OH}}$ are RF power, $\mathrm{RH}$ power and $\mathrm{OH}$ power, respectively. The $\mathrm{RH}$ power is defined as $P_{i}^{\mathrm{RF}} / \mu_{\Sigma}=P_{i}^{\mathrm{RH}}$, where $\mu_{\Sigma}$ is the RH efficiency [20]. The throughput of the system is defined as $R_{\mathrm{AP}, i}=M / T_{\mathrm{AP}}^{\mathrm{RH}, i}$, which is greater or equal to the offered load: $R_{\text {traffic }}=M / T_{\mathrm{AP}}^{\mathrm{OH}}$. The term $\frac{P_{i}^{\mathrm{RH}}}{R_{\mathrm{AP}, i}}$ in (8) is an indication of the average radio transmission efficiency, which does not consider the $\mathrm{OH}$ energy. This is commonly used to 
measure energy consumption in literature, and is known as the energy-consumption-ratio (ECR) [21]. $n$ refers to the total number of APs.

For a given offered load demanded by users, a more spectral efficient deployment is able to transmit the same data for a short transmission time. Over time, this amounts to a reduction of the $\mathrm{RH}$ energy consumption. The energy saving caused by spectral efficiency alone is upper-bounded (ERG threshold) by the ratio of $\mathrm{OH}$ to OP. This upperbound $\left(\mathrm{ERG}_{\mathrm{RANupper}-\text { bound }}^{\mathrm{OP}}=\frac{P_{\text {test }}^{\mathrm{OH}}}{P_{\text {ref. }}^{\mathrm{RH}}+P_{\text {ref. }}^{\mathrm{OH}}}=\frac{P_{\text {test }}^{\mathrm{OH}}}{P_{\text {ref. }}^{\mathrm{OP}}}\right)$ can be obtained when $R_{\mathrm{AP}, \text { test }}$ in Equation (8) approaches infinity on the condition that the same number of APs deployed for both reference and test systems is considered. In order to significantly reduce energy consumption further, a reduction in the number of APs is required to meet the QoS needed. This can only be accomplished by significantly improving the overall throughput of the AP deployment. This article proposes novel location optimisation simulation and theoretical results to achieve this.

\section{Simulation and theoretical results}

\subsection{Single room AP number}

\subsubsection{Conventional scenario}

In the single room scenario, the article has investigated the maximum downlink user QoS achieved by at least $95 \%$ of the users and the average user data rate. This was done by the simulation only. A total number of 50 users are distributed randomly and uniformly across the whole indoor room whose area is $20 \mathrm{~m} \times 16 \mathrm{~m}$. Only one single floor building without light internal walls (e.g. plaster board) is considered. Due to the omni-directional radiation pattern of the AP, its deployment was conducted to minimise the mean distance from users to AP. Therefore, there is always one AP deployed in the middle of the room except for the case of two APs, in which case they are placed at the foci of the ellipse layout. For the remaining deployments, all other APs excluding the middle one are placed evenly around the circumference of the ellipse as shown in Figure 3.

Investigation of optimal FAP placement and interference management will be described in the next sub-section. It is shown that 1 FAP can achieve the highest user QoS and average user data rate of just over 1 Mbit/ $\mathrm{s}$ with SISO deployment owing to the absence of any interference. It is worth mentioning that there is a $43 \%$ improvement in the spectral efficiency when using 1 FAP as compared to $1802.11 \mathrm{n} \mathrm{AP}$ as shown in Figure 4a. This gain is due to the different scheduler mechanism as well as the link level MCS between LTE-femtocell and $802.11 \mathrm{n}$ network. For the same bandwidth, LTE-femtocell employs a more spectral efficient adaptive MCS than 802.11n. 1 FAP offers 4.44\% ERG against 1 baseline 802.11n AP with SISO deployment. This is shown in Figure 4d. It was found that for a single AP deployment, a single FAP is more spectral and energy efficient than a single 802.11n AP.

As the number of APs is increased, the $802.11 \mathrm{n}$ deployment is always more spectrally and energy efficient due to the increased operating bandwidth of 60 $\mathrm{MHz}$ with frequency reuse pattern 3, compared to the LTE bandwidth of $20 \mathrm{MHz}$ with frequency reuse pattern 1. As the number of 802.11n APs increases to beyond 3, the interference that arises between the APs will cause a degradation of overall downlink user QoS and average user data rate performance. For the number of APs

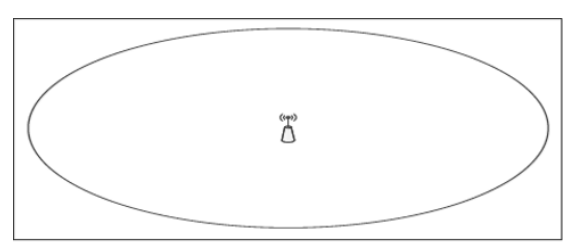

(a) $1 \mathrm{AP}$

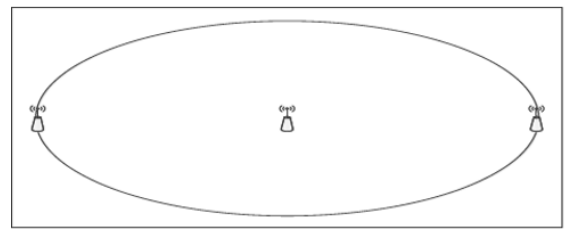

(c) $3 \mathrm{APs}$

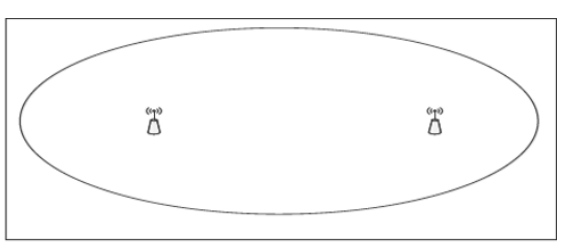

(b) 2 APs

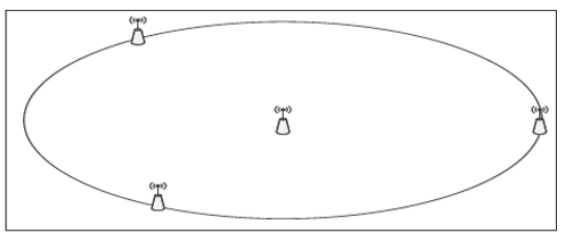

(d) $4 \mathrm{APs}$

Figure 3 1, 2, 3 and 4 APs placement. 
greater than 2, 802.11 APs provides 2.61 to $21.80 \%$ ERG over the FAP deployment. The results are shown in Figure $4 \mathrm{~b}, \mathrm{~d}$.

Therefore, for a single AP deployment, an LTE FAP is more spectrally and energy efficient than an 802.11n AP. This is true both with and without a fully loaded micro-cell interference source. In order to achieve a higher user QoS performance, deploying more 802.11n APs is the more spectrally and energy efficient. No more than 3 802.11n APs should be deployed in the same room; any more causes mutual interference and degrades the aggregate QoS received by the users.

\subsubsection{Alternative scenario}

The alternative scenario is defined in the body of investigation, in which both FAPs and 802.11n APs have a total bandwidth of $20 \mathrm{MHz}$ with different frequency reuse pattern 1 and 3, respectively. The result of 1 FAP and $1802.11 \mathrm{n}$ AP for both conventional and alternative scenarios are identical as shown in Figure 4a. Figure 4c,d, average user data rate and ERG performance for 1 FAP and 3 FAPs in both conventional and alternative scenario with a baseline 802.11n network. In the alternative scenario, FAP outperforms 802.11 AP when the number of APs is 3. The average user data rate for three FAPs is $1.12 \mathrm{Mbit} / \mathrm{s}$ while this value for $3802.11 \mathrm{APs}$ is $0.22 \mathrm{Mbit} / \mathrm{s}$. This is because 802.11 AP suffers server interference from other APs in the alternative scenario and its PHY adopts convolution codes which is less efficient than turbo codes used in LTE-femtocell. Figure $4 \mathrm{~d}$ indicates three FAPs provides an ERG of $20.08 \%$ in alternative scenario while $3802.11 \mathrm{n}$ APs offers an ERG of $21.80 \%$ in conventional scenario. Hence FAP investigation is particular interest in the following sections.

\subsubsection{Remarks}

The results in Figure 4a covers the results of all four possible combinations of comparison between one FAP and one $802.11 \mathrm{n} \mathrm{AP}$ in either conventional or alternative scenario while the results in Figure 4c contains the same number of combinations results for the case of 3

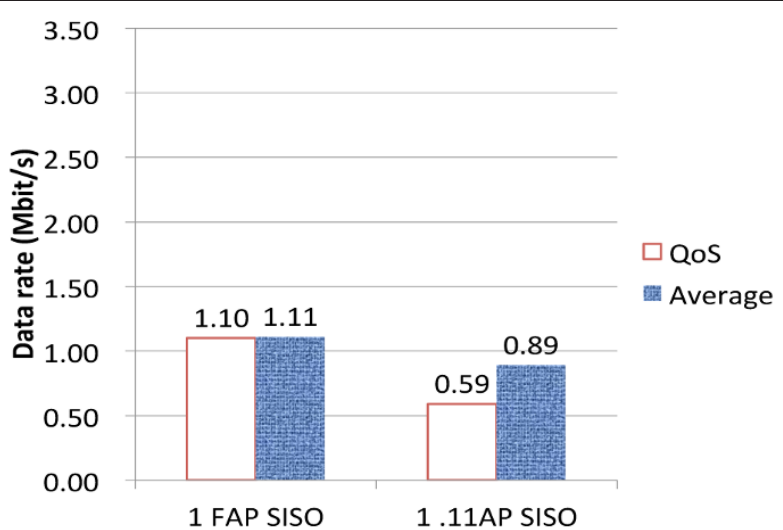

(a) 1 FAP vs. $1802.11 \mathrm{n} \mathrm{AP}($ Conventional $)$

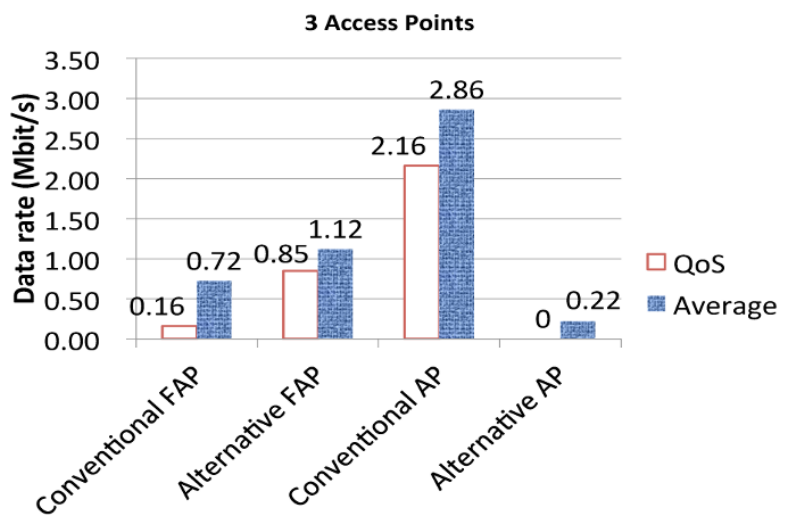

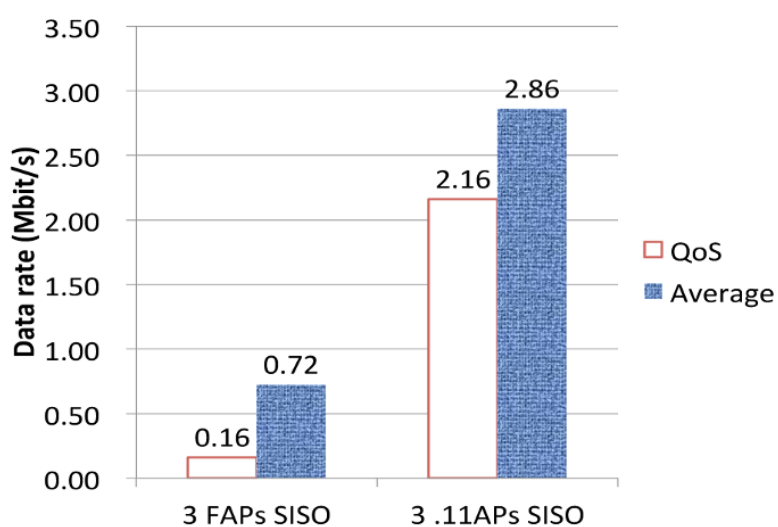

(b) 3 FAPs vs. $3802.11 \mathrm{n}$ APs (Conventional)

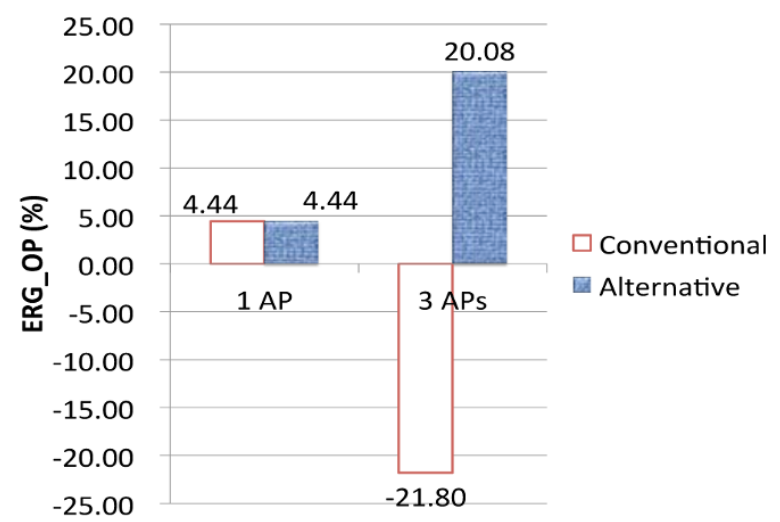

(c) 3 FAPs vs. 3 802.11n APs (Conventional vs. Alternative) (d) ERG comparison for conventional and alternative scenarios

Figure 4 QoS, average user data rate and ERG comparison between LTE-femtocells and $802.11 \mathrm{n}$ with SISO deployment without outdoor interference in both conventional and alternative scenarios. 
FAPs and 3 802.11n AP. These four possible combinations are conventional FAP versus alternative AP and conventional AP versus alternative FAP besides the other two which have already been covered in the above sections. It is worth mentioning that 3 FAPs in conventional scenario is more energy efficient than 3 APs in alternative scenario. This is due to the mutual impact from the the different scheduler mechanism and coding scheme applied in both systems.

\subsection{Single room AP placement}

Previously, the optimal number of APs to deploy in a single room has been considered. The conclusion was that for a low QoS target, 1 FAP is the most energy efficient deployment. For higher user QoS targets, 2-3 802.11n APs should be deployed. Next, simulation is used to determine where to place the 1 FAP given that there is an outdoor interference source from a microcell, and where to place 2-6 co-frequency FAPs that interfere with each other. Furthermore, result of 1 FAP with a theoretical background has been reinforced, which can be found in the Appendix of this article.

\subsubsection{FAP}

The optimal placement of 1 FAP is judged according to the strength of the outdoor micro-cell source. Another baseline FAP deployment has been considered for comparison. In this baseline scenario, FAP is placed at the corner of the room where the power socket is typically located.

The expression of the mean capacity of the femtocell network with respect to the position of the FAP $b$ can be expressed as:

$$
\bar{C}^{b}= \begin{cases}P_{1}+Q_{1}+R_{1}-\frac{\alpha}{Y}\left(T_{1}-U_{1}\right), & 0<b \leq b_{1} \\ P_{2}+Q_{2}+R_{1}+R_{2}-\frac{\alpha}{Y}\left(T_{1}+T_{2}-U_{1}-U_{2}\right), & b_{1}<b \leq b_{2} \\ P_{3}+Q_{3}+R_{2}-\frac{\alpha}{Y}\left(T_{2}-U_{2}\right), & b_{2}<b<Y\end{cases}
$$

where $\quad P_{1}=\frac{\left(b+d_{b p 2}\right) C_{\mathrm{s}}}{Y}, \quad Q_{1}=\frac{\left(Y-b-d_{b p 2}\right) \log _{2} K_{\gamma}}{Y}$, $R_{1}=\frac{\beta \log _{2} 10}{2 Y}\left[Y^{2}-\left(b+d_{b p 2}\right)^{2}\right], \alpha=1.87$ and $Y$ is the length of the room. $T_{1}=Y \log _{2}(Y-b)-\left(b+d_{b p 2}\right) \log _{2} d_{b p 2}$, $U_{1}=\left(\frac{Y-b-d_{b p 2}}{\ln 2}+b \log _{2} \frac{Y-b}{d_{b p 2}}\right) . \quad C_{\mathrm{s}} \quad$ in $\quad P_{1}$ equals $\log _{2}\left(1+10^{\frac{\gamma s}{10}}\right) \mathrm{bit} / \mathrm{s} / \mathrm{Hz}$ where $\gamma_{s}$ is the saturation SNR threshold. $\quad P_{2}=\frac{\left(d_{b p 1}+d_{b p 2}\right) C_{\mathrm{s}}}{Y}, \quad Q_{2}=\frac{\left(Y-d_{b p 1}-d_{b p 2}\right) \log _{2} K_{\gamma}}{Y}$, $R_{2}=\frac{\beta \log _{2} 10}{2 Y}\left(b-d_{b p 1}\right)^{2}, \quad T_{2}=\left(b-d_{b p 1}\right) \log _{2} \quad d_{b p 1} \quad$ and $U_{2}=\frac{b-d_{b p 1}}{\ln 2}+b \log _{2} \frac{d_{b p 1}}{b} . \quad K_{\gamma} \quad$ in $\quad Q_{2}$ is $\frac{P_{\mathrm{FAP}} D^{3.67} \times 10^{\left(22.7+\mathrm{PL}_{\text {wall }} / 10\right.} \times\left(\frac{f}{5}\right)^{2.6}}{P_{\text {micro }} \bar{G}_{\text {micro }} \times 10^{46.8 / 10} \times\left(\frac{f}{5}\right)^{2}}$, where $P_{\mathrm{FAP}}$ and $P_{\text {micro }}$ are the transmitting power of FAP and micro-cell station, respectively. $\bar{G}_{\text {micro }}$ is the expected value of the antenna gain from the micro-cell BS. $f$ is the operation frequency. $d_{b p 1}=B \exp \left[-W(F)\right.$ and $d_{b p 2}=B \exp [-W$
$(-F)$ ] where $B=10 \frac{b}{20 \alpha}\left(\frac{K \gamma}{10 \frac{\gamma s}{10}}\right)^{\frac{1}{\alpha}}, F=\frac{\ln 10}{20 \alpha} B, W$ is Lambert $W$ function. Finally, $P_{3}=\frac{\left(Y-b+d_{b p 1}\right) C_{\mathrm{s}}}{Y}$, $b_{1}=\sqrt[\alpha]{\frac{K_{\gamma}}{\frac{\gamma_{s}}{10}}}, b_{1}=\sqrt[\alpha]{\frac{K_{\gamma}}{\frac{\gamma_{s}}{10}}}$ and $b_{2}=Y-\sqrt[\alpha]{\frac{K_{\gamma} 10^{\beta Y}}{10^{\frac{\gamma_{s}}{10}}}}$.

It can be shown that the function is convex and that according to the first rule of finding the maximum value of a function, stationary points can be determined by differentiating Equation (Note : $\frac{\partial d_{b p 1}}{\partial b}=\frac{B \exp [-W(F)] \ln 10}{20 \alpha[1+W(F)]}$ and $\frac{\partial d_{b p 2}}{\partial b}=\frac{B \exp [-W(-F)] \ln 10}{20 \alpha[1+W(-F)]}$ ) and then solving the differentiated function for zeros. The resulting expression is a closed form expression, but is unfortunately too long for the scope of this article. All the stationary points are tested in order to verify the type of the stationary points (max) by checking if the corresponding value in the second-order differential function of Equation (9) is negative. Finally, the mean capacity value(s) corresponding to all the stationary points are compared with all endpoints of the interval of each sub-function in Equation (9) and the global maximum value is selected as the maximum of mean capacity. The solution $b_{\text {opt }}$ is the optimal coordinate for FAP placement. Detailed derivations of Equation (9) can be found in the Appendix.

The result of the mean capacity difference between the optimal and the conventional is illustrated in the sub-plots of Figure $5 \mathrm{a}$. The theoretical results are shown in lines and are compared with the simulation results shown as symbols. The parameters used in the investigation are as follows: office size $(10 \mathrm{~m} \times 20 \mathrm{~m})$, system bandwidth $(20 \mathrm{MHz})$, carrier frequency $(2,130 \mathrm{MHz})$, total number of users (10), sub-carriers per Physical Resource Block (12), FAP transmitting power (0.1 W), micro-cell transmitting power $(20 \mathrm{~W})$, FAP RH efficiency $(6.67 \%)$, FAP OH power $(5.2 \mathrm{~W})$, micro-cell distance away from the room (150-450 m), and Wall loss (10 dB).

Figure 5b shows the OP ERG performance. The result in line is calculated based on the throughput from the theory while the result in symbols is obtained based on the throughput from the simulation. They were both obtained from the Equation (8). The results in Figure 5 show that 1 FAP should be located between the middle of the room to the wall closest to the outdoor interference source. As the strength of the micro-cell interference decreases due to it being either further away, stronger wall loss, or lower transmitting power, the FAP should be moved closer to the wall. This is because most of the room is in capacity saturation, and the FAP should be moved to compensate for regions which are not. It is important to consider capacity saturation, 

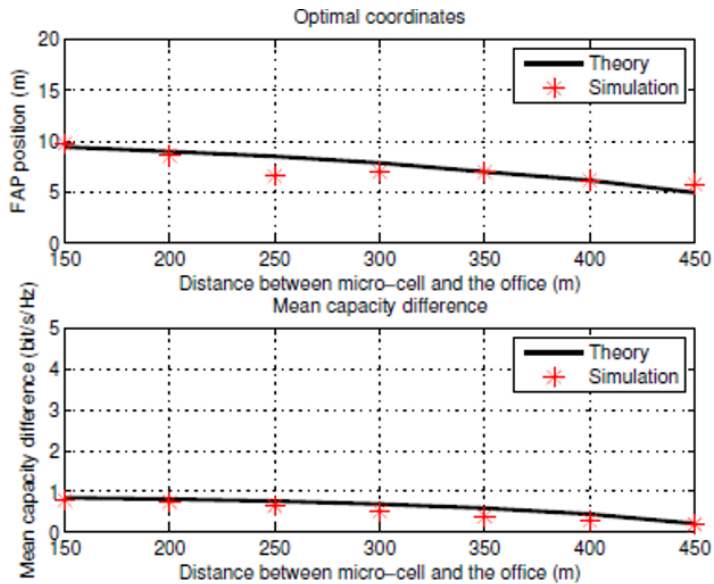

(a) Optimal position and mean capacity difference vs. micro-cell-to-room distance

Figure 51 FAP optimal location and ERG performance with respect to outdoor micro-cell interference

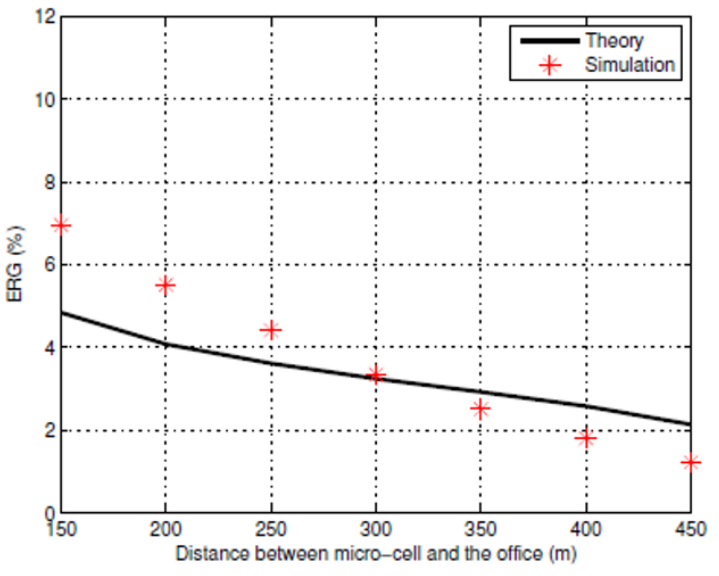

(b) ERG vs. micro-cell-to-room distance without which the FAP's optimal location is always likely to be in centre of the room.

\subsubsection{2-6 FAPs}

The results in Figure 6 shows that for more than one cofrequency AP is deployed in the same room, the mutual interference between them dominate. The meaning of dots in different colours in the plots is the association of users to different FAPs. Their location is a trade-off between: being in the central area to reduce path-loss distance to the users, and being further away from each other to reduce mutual interference. The RAN throughput for both optimal and conventional scenario increases as more FAPs are deployed. It reaches saturation region when 6 FAPs are placed in optimal approach as shown in Figure 7a. Compared to the baseline elliptical deployment, there is an average 6\% ERG obtained from the optimal deployment shown in Figure 7b.

\subsection{Multi-room multi-floor FAP placement}

The article now considers a building with $F$ floors $R$ rooms per floor. The framework of this comparison is how much energy is saved when the location of FAP is optimised compared to an even distribution of FAPs across the building. This investigation is done for FAPs in the presence of an outdoor micro-cell interference source. A series of RAN QoS offered loads on the system is considered and what the minimum number of FAPs is required to meet this targeted load is examined. The parameters used in the investigation are as follows: number of floors (3), number of rooms per floor (9), room size $(10 \mathrm{~m} \times 20 \mathrm{~m} \times 4 \mathrm{~m})$, system bandwidth $(20 \mathrm{MHz})$, carrier frequency $(2130 \mathrm{MHz})$, total number of users per building (300), sub-carriers per Physical Resource Block (12), FAP transmitting power $(0.1 \mathrm{~W})$, micro-cell transmitting power $(20 \mathrm{~W})$, FAP RH efficiency $(6.67 \%)$, FAP OH power $(5.2 \mathrm{~W})$, micro-cell distance away from the room $(200 \mathrm{~m})$, and wall loss $(10 \mathrm{~dB})$. Figure 8 shows the optimal locations of FAPs for different number of FAPs required and the associated capacity and energy consumption improvements. The rooms on the top floor has been numbered as 1-9 followed by the rooms on the middle floor (1018 ) and the ones on the ground floor (19-27). the micro-cell BS is located close to the side of room numbered (7-9, 16-18 and 25-27). FAP positions in yellow represent that the system performance will be almost same by deploying FAP on either of these positions. FAP positions in blue are the recommended optimal ones in each scenario.

The results in Figure 8a shows that at least 1-2 FAP(s) are always required near the wall that faces the outdoor interference source, and this should be on the floor with a similar height to the height of the interfering microcell-site. The other FAPs should be deployed on other floors at the far corners in alternating pattern to minimise the interference. The positions of the FAPs in blue have to be fixed while one of the FAPs in yellow can be selected as the last FAP position. A design principle can be summarised as follows:

(1) In the presence of no strong outdoor interference, deploy a single FAP at centre of building. In the presence of outdoor micro-cell interference, deploy the FAP near the wall that is closest to the 


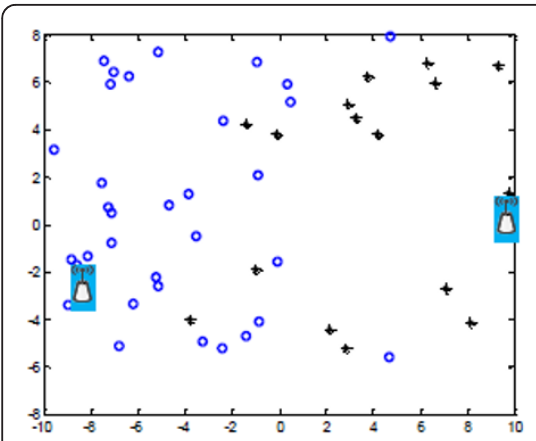

(a) 2 FAPs

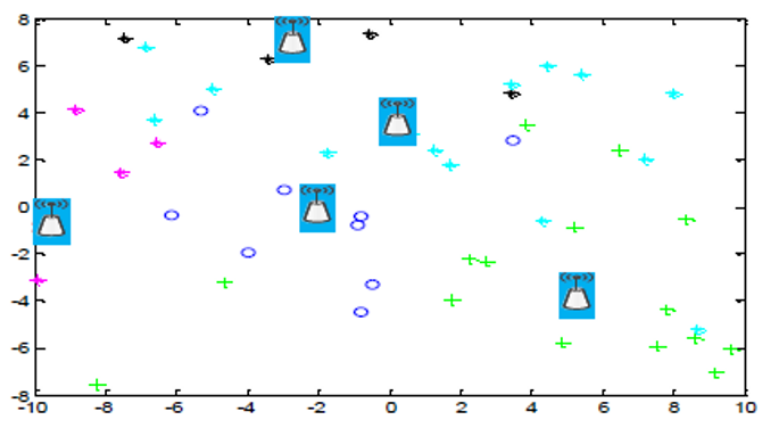

(d) 5 FAPs
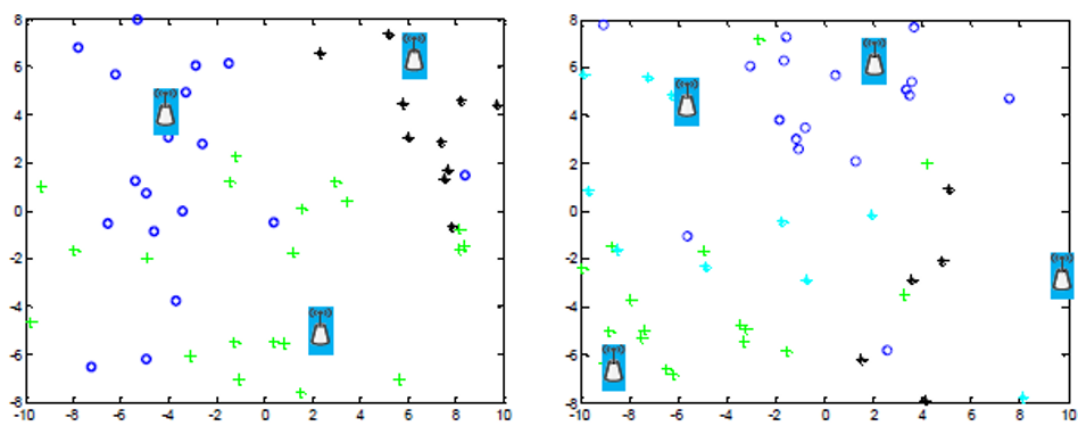

(b) 3 FAPs (c) 4 FAPs

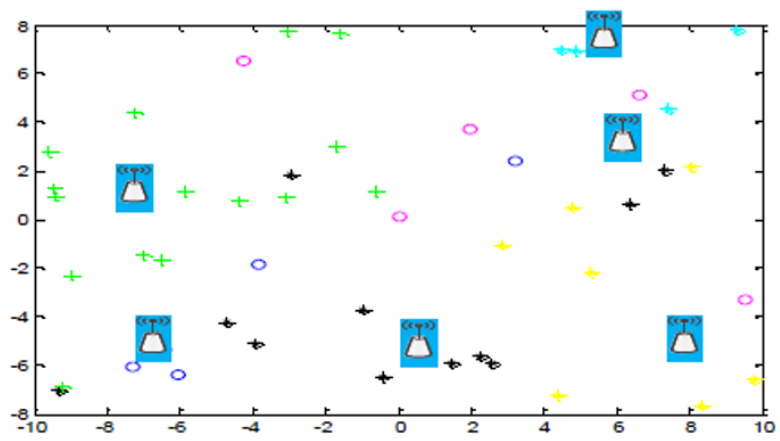

(e) 6 FAPs

Figure 62 to 6 FAPs optimal location with respect to mutual interference.

outdoor interference source. The floor level should be one that is closest to the height of the micro-cell.

(2) Any single additional FAP should be deployed also near the aforementioned wall on the same floor, but not in the same room as the first FAP.

(3) Any multiple additional FAPs should be deployed not on the same floor, and at the opposite side of the building in corner rooms. These FAPs should not be on the same floor as FAPs placed in Steps 1 and 2 and with each other in Step 3.

(4) Any additional FAPs that do not satisfy rule 3. is likely to cause energy inefficiency.

Generally speaking, this rule can cover the optimisation of FAP placement for up to 6 FAPs, which can provide a sufficiently high QoS. The RAN QoS increases as the number of FAPs increases. This optimal deployment offers an average $12 \%$ ERG compared to the baseline even distributional deployment. This is shown in Figure 8b. Figure $8 \mathrm{c}$ illustrates that how much energy can be reduced while deploying the optimal FAPs in this building when comparing to the baseline scenario for a certain RAN QoS. As the number of FAPs needed for different targeted RAN QoS is not always same for optimal and baseline deployment, ERG threshold is waived is this comparison.

It can be noted that the solution of optimising the FAP location does not significantly degrade the outdoor network performance. By moving the FAP from centre to a point that is closer to the outdoor interference source, the interference from the FAP to the outside network is increased by up to $2.5 \mathrm{~dB}$. Given that the outdoor interference is more dominated by outdoor interference from other micro-cells the total interference is not significantly increased. This is to say that the interference generated by the FAP to outdoor users is not significantly increased.

\section{Discussions}

The key conclusions are as follows:

- Deploying one FAP is always more spectral and energy efficient than one 802.11n Wi-Fi AP;

- Deploying up to 3 multiple 802.11n APs is always more energy efficient than deploying multiple FAPs within the same building in conventional scenario. For buildings with more than one room, no APs 


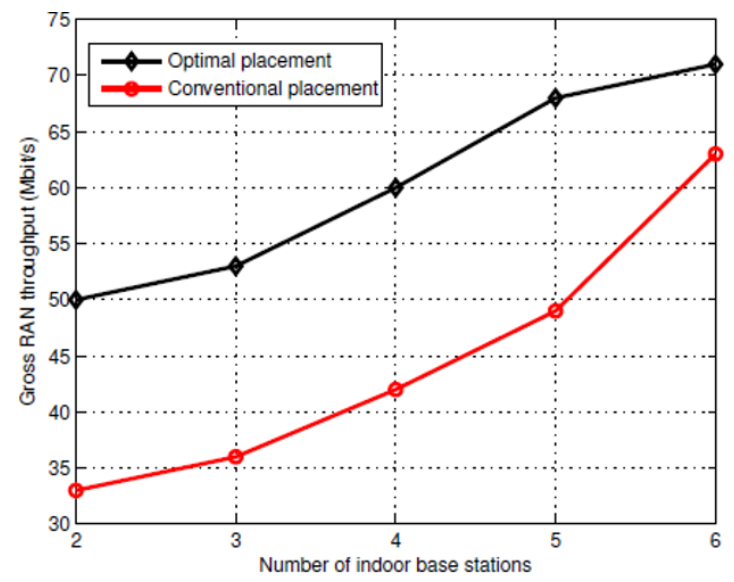

(a) Average RAN throughput vs. number of FAPs

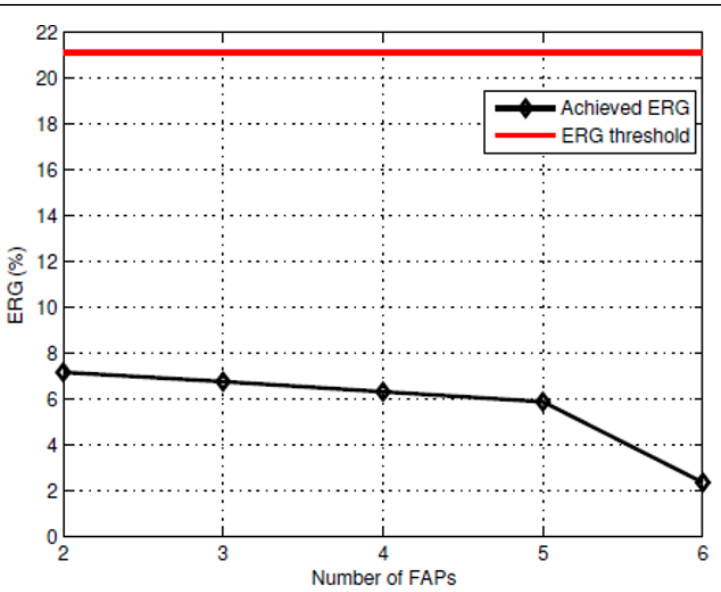

(b) ERG vs. number of FAPs

Figure 7 ERG performance for 2 to 6 FAPs between optimal and conventional deployment.

should be deployed in the same room of a building; - In the presence of a strong outdoor interference source (i.e., from a micro-cell), the location of some of the co-frequency FAPs, should be placed near the wall facing the interference source to counter-act the high level of indoor interference.

The energy reductions for several key comparisons are as follows:

- A single FAP versus A single 802.11n AP saves 4.44\% OP ERG;

- Three $802.11 \mathrm{n}$ APs versus three FAPs saves

$21.80 \%$ OP ERG in conventional scenario;

- Three FAPs versus three $802.11 \mathrm{n}$ APs saves 20.08\% OP ERG in alternative scenario;

- Optimising placement of a single FAP in a single room saves 5\% OP ERG;

- Optimising placement of multiple FAPs in a multi-room building saves average 12\% OP ERG.

In general, improving the location of the FAPs, whilst keeping the number the same can improve the RAN throughput by $33 \%$. Whilst, improving the location of the FAPs and reducing the number of FAPs can save $40 \%$ energy.

\section{Conclusions}

This body of investigation has proposed a novel strategy for indoor AP deployment for both LTE-Femtocells and $802.11 \mathrm{n} \mathrm{Wi-Fi} \mathrm{multiple} \mathrm{access} \mathrm{technologies.} \mathrm{This} \mathrm{is}$ done for a single room and multiple room scenarios. Our comparisons show that between 5 and 40\% ERG can be saved by optimising the AP number and location with respect to the propagation parameters and interference scenario. Our baseline simulation results are reinforced with a novel theoretical framework, which can be used to develop and optimise indoor deployment while causing very little SINR degradation for micro-cell users.

\section{Methods}

The analysis in this paper is conducted by using both Monte-Carlo computation in a MAT-LAB software environment and theoretical expressions.

\section{Appendix \\ Theoretical framework of 1 FAP optimisation Introduction}

In order to verify the simulation result of the optimal coordinate for a FAP to maximise the mean throughput in a particular indoor room, an extensive theoretical model to solve this optimal problem has been developed under certain assumptions. Those assumptions are as follows:

- The throughput experienced by indoor users are always in the high SINR regime, and that at least some region of the room experiences saturated throughput. This has been verified to be accurate after extensive simulation runs.

- The variation of throughput is mainly along the axis of the FAP-Microcell, and not orthogonal to it.

- A modified Shannon expression is used for throughput, which accounts for the mutual information saturation of modulation and coding schemes. 


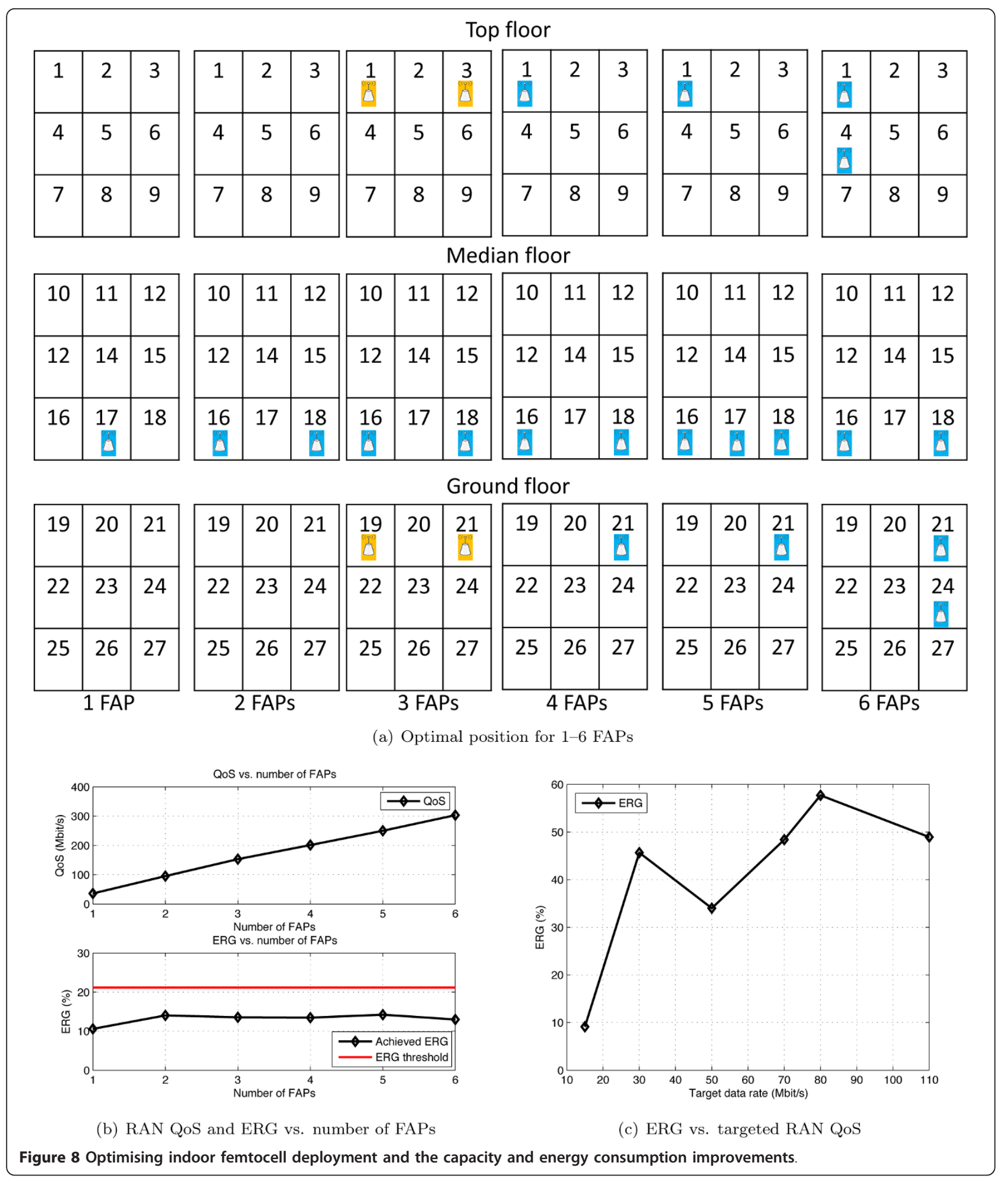


In a rectangular room with Cartesian co-ordinates of $x$ and $y, \gamma_{x, y}$ is defined as the received SINR for a user at a particular position can be expressed in (10).

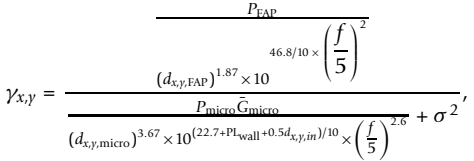

$$
\begin{aligned}
& \approx \frac{P_{\mathrm{FAP}} D^{3.67} \times 10^{\left(22.7+\mathrm{PL}_{\text {wat1 }}\right) / 10} \times\left(\frac{f}{5}\right)^{2.6}}{P_{\text {micro }} \bar{G}_{\text {micro }} \times 10^{46.8 / 10 \times\left(\frac{f}{5}\right)^{2}}} \frac{10^{0.5 d_{x, y \text { in }} / 10}}{d_{x, y, \mathrm{FAP}}^{1.87}}=K_{\gamma} \frac{10^{\beta d_{x, y, \mathrm{in}}}}{d_{i, \mathrm{FAP}}^{\alpha}}, \\
& \text { Where } \frac{P_{\mathrm{FAP}} D^{3.67} \times 10^{\left(22.7+\mathrm{PL}_{\text {wall }}\right) / 10} \times\left(\frac{f}{5}\right)^{2.6}}{P_{\text {micro }} \bar{G}_{\text {micro }} \times 10^{46.8 / 10} \times\left(\frac{f}{5}\right)^{2}} \text { is denoted as } K_{\gamma} \alpha
\end{aligned}
$$

$=1.87$ and $\beta=0.05$. $P_{\mathrm{FAP}}$ and $P_{\text {micro }}$ are the transmitting power of FAP and micro-cell station, respectively. $\bar{G}_{\text {micro }}$ is the expected value of the antenna gain from the micro-cell BS. $d_{x, y \text {,FAP }}$ is the $i$ th FAP-to-grid distance and $d_{x, y, \text { micro }}$ is the $i$ th micro-cell-to-grid distance. $d_{x, y, \text { in }}$ is the distance between the wall and $i^{\text {th }}$ grid. As $D \gg d_{x}$ $y$,in,$d_{x, y, \text { micro }}$ can be accurately estimated as $D . \gamma_{x, y}$ can then be re-written as $K_{\gamma} \frac{10^{\beta d_{x, y, \text { in }}}}{d_{i \text {, AP }}^{\alpha}}$.

For a given FAP position of $(x=a, y=b)$, the mean capacity of users attached to this FAP is therefore:

$$
\bar{C}_{a, b} \approx \frac{1}{A} \iint_{A} \log _{2}\left(\gamma_{x, y}\right) \mathrm{d} x \mathrm{~d} y=\frac{1}{A} \iint_{A} \log _{2}\left[K_{\gamma} \frac{10^{\beta|y|}}{\left(\sqrt{(x-a)^{2}+(y-b)^{2}}\right)^{\alpha}}\right] \mathrm{d} x \mathrm{~d} y .
$$

It can be noted that $\gamma_{x, y}(15-45 \mathrm{~dB})$ is much greater than 1 which makes the above high SINR approximation reasonable. According to the MCS simulations, the throughput will saturate when $\gamma_{x, y}>\gamma_{s}=35.94 \mathrm{~dB}$, where $\gamma_{s}$ is the saturation SNR threshold. Hence, $\bar{C}_{a, b}$ should be modified to reflect this saturated region, which is shown as $d_{b p 1}$ and $d_{b p 2}$ in Figure 9. It is also worth pointing out that $\gamma_{x, y}$ hardly fluctuates along $x$ axis outside the saturated region. Equation (11) can be re-written as Equation (12) under the condition that the $x$ coordinates of all grids are independent of $\gamma_{x, y}$ by using Wyner [22] Model:

$$
\bar{C}^{b}=C_{\mathrm{s}} Y_{\mathrm{s}}+\frac{1}{Y_{\mathrm{ns}}} \int_{Y_{\mathrm{ns}}} \log _{2}\left(K_{\gamma} \frac{10^{\beta \gamma}}{|\gamma-b|^{\alpha}}\right) \mathrm{d} y,
$$

where $C_{\mathrm{s}}=\log _{2}\left(1+10^{\frac{\gamma_{\mathrm{s}}}{10}}\right) \mathrm{bit} / \mathrm{s} / \mathrm{HZ} . Y_{\mathrm{s}}$ and $Y_{\mathrm{ns}}$ are the length of saturated and non-saturated regions, respectively. Equation (12) can be split into three subfunctions with respect to FAP position $b$.

It is important to note that due to capacity saturation, the mean capacity of the indoor users has to be broken up into the following 3 scenarios:
(1) Scenario 1: the FAP is close to the window $(0<b \leq$ $b_{1}$ ) so that the only non-saturated capacity region is away towards the wall.

(2) Scenario 2: the FAP is in the middle region of the room $\left(b_{1}<b \leq b_{2}\right)$ so that the non-saturated capacity regions exist both towards the window and the wall.

(3) Scenario 3: the FAP is close to the far wall $\left(b_{2}<b\right.$ $\leq Y$ ) so that the only non-saturated capacity region is towards the window.

We now consider these scenarios in turn and combine their theoretical formulation.

\section{Formulation Scenario 1}

In the first scenario, the farthest coordinate for FAP cannot exceed $b_{1}$ where the SINR value at the wall close to the micro-cell side (i.e. $d_{x, y \text {,in }}=0$ ) should just achieve the saturation SNR threshold $\gamma_{s}$. Hence, $b_{1}$ can be calculated as:

$$
K_{\gamma} \frac{10^{\beta d_{\gamma, \text { in }}}}{b_{1}^{\alpha}}=10^{\frac{\gamma_{s}}{10}}, \Rightarrow b_{1}=\sqrt[\alpha]{\frac{K_{\gamma}}{10^{\frac{\gamma_{s}}{10}}}}
$$

The capacity can therefore be expressed by:

$$
\begin{aligned}
\bar{C}_{1}^{b}= & \frac{1}{Y} \int_{0}^{b+d_{b p 2}} C_{s} \mathrm{~d} y+\frac{1}{Y} \int_{b+d_{b p 2}}^{Y} \log _{2}\left[K_{\gamma} \frac{10^{\beta \gamma}}{(y-b)^{\alpha}}\right] \mathrm{d} y, \\
= & \underbrace{\frac{\left(b+d_{b p 2}\right) C_{s}}{Y}}_{=P_{1}}+\underbrace{\frac{\left(Y-b-d_{b p 2}\right) \log _{2} K_{\gamma}}{Y}}_{=Q_{1}}+\frac{\beta \log _{2} 10}{Y} \\
=S_{1} & \int_{b+d_{b p 2}}^{Y} y \mathrm{~d} y-\frac{\alpha}{Y} \underbrace{\int_{\log _{2}}(\gamma-b) \mathrm{d} y,}_{b+d_{b p 2}} \\
= & P_{1}+Q_{1}+\underbrace{\frac{\beta \log _{2} 10}{2 Y}\left[Y^{2}-\left(b+d_{b p 2}\right)^{2}\right]-\frac{\alpha}{Y} S_{1}}_{=R_{1}} .
\end{aligned}
$$

The term $d_{b p 2}$ can be solved by the following equation:

$$
K_{\gamma} \frac{10^{\beta\left(b+d_{b p 2}\right)}}{d_{b p 2}^{\alpha}}=10^{\frac{\gamma_{S}}{10}}, \Rightarrow d_{b p 2}=B \exp [-W(-F)]
$$

where $B=10^{\frac{b}{20 \alpha}}\left(\frac{K_{\gamma}}{\frac{\gamma_{S}}{10}}\right)^{\frac{1}{\alpha}}, F=\frac{\ln 10}{20 \alpha} B, W$ is Lambert

$W$ function, namely the branches of the inverse relation of the function $f(W)=W \exp (W)$. $S_{1}$ can then be calculated in Equation (16): 


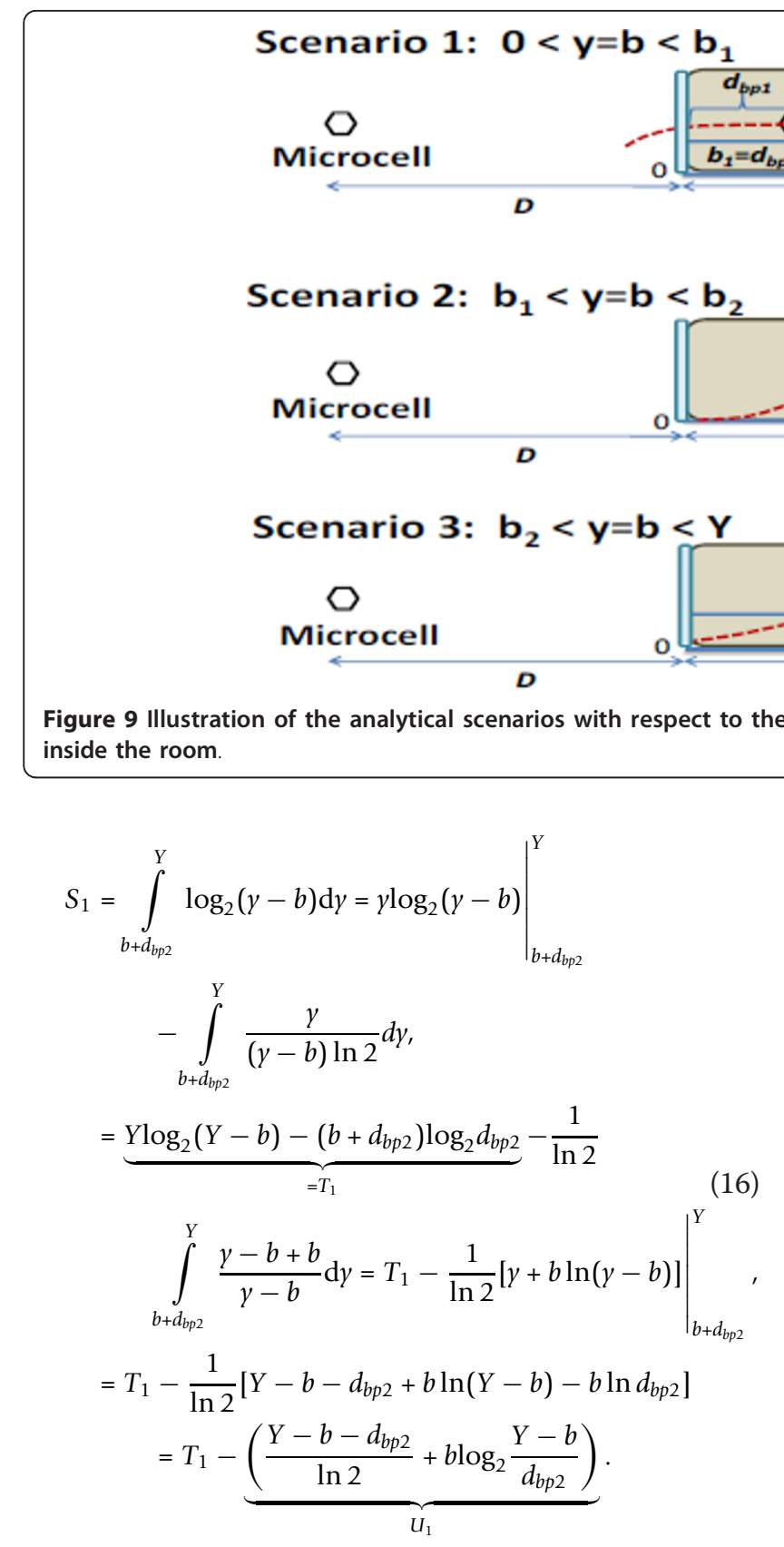

By substituting Equation (16) for $S_{1}$ in Equation (14), $\bar{C}_{1}^{b}$ can be finalised as:

$$
\bar{C}_{1}^{b}=P_{1}+Q_{1}+R_{1}-\frac{\alpha}{Y}\left(T_{1}-U_{1}\right) .
$$

\section{Formulation Scenario 2}

When the position of FAP is on the coordinate $b_{2}$, the user's SINR at the other end of the room is equal to $\gamma_{s}$. $b_{2}$ needs to be determined before assigning the proper limits for the mean capacity integral. With reference to Equation (13), $b_{2}$ can be found to be:

$$
b_{2}=Y-\sqrt[\alpha]{\frac{K_{\gamma} 10^{\beta Y}}{10^{\frac{\gamma_{s}}{10}}}} .
$$

After $b_{2}$ is solved, the mean capacity in this scenario can therefore be expressed by:

$$
\begin{aligned}
\bar{C}_{2}^{b} & =\frac{1}{Y} \int_{0}^{b-d_{p p 1}} \log _{2}\left[K_{y} \frac{10^{\beta y}}{(b-\gamma)^{\alpha}}\right] \mathrm{d} y+\frac{1}{Y} \int_{b-d_{p+1}}^{b+d_{d_{p 2}}} C_{s} \mathrm{~d} y+\frac{1}{Y} \int_{b+d_{d_{p 2}}}^{Y} \log _{2}\left[K_{\gamma} \frac{10^{\beta y}}{(y-b)^{\alpha}}\right] \mathrm{d} y \\
& =P_{2}+Q_{2}+R_{1}+R_{2}-\frac{\alpha}{Y}\left(T_{1}+T_{2}-U_{1}-U_{2}\right),
\end{aligned}
$$

where $d_{b p 1}$ can be solved by the following equation:

$$
\begin{array}{cc}
K_{\gamma} \frac{10^{\beta\left(b-d_{b p 1}\right)}}{d_{b p 1}^{\alpha}}=10 \frac{\gamma_{s}}{10}, \quad \Rightarrow d_{b p 1}=B \exp [-W(F)], \quad(20) \\
P_{2}=\frac{\left(d_{b p 1}+d_{b p 2}\right) C_{s}}{Y}, & Q_{2}=\frac{\left(Y-d_{b p 1}-d_{b p 2}\right) \log _{2} K_{\gamma}}{Y}, \\
T_{2}=\left(b-d_{b p 1}\right) \log _{2} d_{b p 1}, & T_{2}=\left(b-d_{b p 1}\right) \log _{2} d_{b p 1}, \quad \text { and } \\
U_{2}=\frac{b-d_{b p 1}}{\ln 2}+b \log _{2} \frac{d_{b p 1}}{b} . &
\end{array}
$$

\section{Formulation Scenario 3}

In the last scenario, the mean capacity expression is very similar to that in scenario one and is given by:

$$
\begin{aligned}
\bar{C}_{3}^{b} & =\frac{1}{Y} \int_{0}^{b-d_{b p 1}} \log _{2}\left[K_{\gamma} \frac{10^{\beta \gamma}}{(b-\gamma)^{\alpha}}\right] \mathrm{d} \gamma \\
& +\frac{1}{Y} \int_{b-d_{b p 1}}^{Y} C_{s} \mathrm{~d} \gamma=P_{3}+Q_{3}+R_{2}-\frac{\alpha}{Y}\left(T_{2}-U_{2}\right),
\end{aligned}
$$

where $P_{3}=\frac{\left(Y-b+d_{b p 1}\right) C_{s}}{Y}$, and $Q_{3}=\frac{\left(b-d_{b p 1}\right) \log _{2} K_{\gamma}}{Y}$. Equations (14), (19) and (21) complete the expression of the 
mean capacity of the network with respect to the position of the FAP $b$ as shown in Equation (9).

\section{Acknowledgements}

The work reported in this paper has formed part of the Green Radio Core 5 Research Programme of the Virtual Centre of Excellence in Mobile and Personal Communications, Mobile VCE. Fully detailed technical reports on this research are available to Industrial Members of the Mobile VCE. http:// www.mobilevce.com

\section{Competing interests}

The authors declare that they have no competing interests.

Received: 1 October 2011 Accepted: 11 June 2012

Published: 11 June 2012

\section{References}

1. V Chandrasekhar, J Andrews, A Gatherer, Femtocell networks: a survey. Communications Magazine, IEEE. 46(9), 59-67 (2008)

2. SF Hasan, NH Siddique, S Chakraborty, Femtocell versus WiFi-A survey and comparison of architecture and performance, in Wireless Communication, Vehicular Technology, Information Theory and Aerospace \& Electronic Systems Technology, 2009. Wireless VITAE 2009. 1st International Conference on, Aalborg, Denmark, IEEE, pp. 916-920 (2009)

3. S Al-Rubaye, A Al-Dulaimi, J Cosmas, Cognitive Femtocell. Vehicular Technology Magazine, IEEE. 6, 44-51 (2011)

4. CH Ko, HY Wei, On-Demand Resource Sharing Mechanism Design in TwoTier OFDMA Femtocell Networks. Vehicular Technology, IEEE Transactions on , 99: 1 (2011)

5. S Park, W Seo, S Choi, D Hong, A Beamforming Codebook Restriction for Cross-Tier Interference Coordination in Two-tier Femtocell Networks. Vehicular Technology, IEEE Transactions on , 99: 1-1 (2011)

6. V Chandrasekhar, JG Andrews, T Muharemovic, Z Shen, A Gatherer, Power control in two-tier femtocell networks. Wireless Communications, IEEE Transactions on. 8(8), 4316-4328 (2009)

7. HS Jo, C Mun, J Moon, JG Yook, Interference mitigation using uplink power control for two-tier femtocell networks. Wireless Communications, IEEE Transactions on. 8(10), 4906-4910 (2009)

8. V Chandrasekhar, J Andrews, Uplink capacity and interference avoidance for two-tier femtocell networks. Wireless Communications, IEEE Transactions on. 8(7), 3498-3509 (2009)

9. D Fagen, PA Vicharelli, J Weitzen, Automated wireless coverage optimization with controlled overlap. Vehicular Technology, IEEE Transactions on. 57(4), 2395-2403 (2008)

10. I Ashraf, H Claussen, LTW Ho, Distributed radio coverage optimization in enterprise femtocell networks, in Communications (ICC), 2010 IEEE International Conference on, Cape Town, South Africa, IEEE, pp.1-6 (2010)

11. JPM Torregoza, R Enkhbat, WJ Hwang, Joint power control, base station assignment, and channel assignment in cognitive femtocell networks. EURASIP Journal on Wireless Communications and Networking. 2010, 6 (2010)

12. S Ki Won, H Harald, ML Stephen, A semianalytical PDF of downlink SINR for femtocell networks. EURASIP Journal on Wireless Communications and Networking. 2010 (2010)

13. M Yavuz, F Meshkati, S Nanda, A Pokhariyal, N Johnson, B Raghothaman, A Richardson, Interference management and performance analysis of UMTS/ HSPA+ femtocells. Communications Magazine, IEEE. 47(9), 102-109 (2009)

14. J Lee, S Bae, Y Kwon, M Chung, Interference Analysis for Femtocell Deployment in OFDMA Systems Based on Fractional Frequency Reuse. Communications Letters, IEEE, 99: 1-3

15. G Bianchi, Performance Analysis of the IEEE 802.11 Distributed Coordination function. IEEE Journal on selected areas in communications. 18(3), 535-547 (2000). doi:10.1109/49.840210

16. YC Tay, KC Chua, A capacity analysis for the IEEE 802.11 MAC protocol. Wireless networks. 7(2), 159-171 (2001). doi:10.1023/A:1016637622896

17. 3GPP, Physical layer aspects for Evolved Universal Terrestrial Radio Access (UTRA). Tech. Rep. TR 25.814 V7.1.0, 3rd Generation Partnership Project (2006)
18. P Kyösti, J Meinilä, L Hentilä, X Zhao, T Jämsä, C Schneider, M Narandzic, M Milojevic, A Hong, J Ylitalo, Others, WINNER II channel models (d1. 1.2 v1. 1). no. IST-4-027756 WINNER II, D1

19. C Mehlführer, M Wrulich, JC Ikuno, D Bosanska, M Rupp, Simulating the Long Term Evolution physical layer, in Proc of the 17th European Signal Processing Conference (EUSIPCO 2009), Glasgow, Scotland, pp. 1471-1478 (2009)

20. EARTH, Energy efficiency analysis of the reference systems, areas of improvements and target breakdown. Tech. Rep. Deliverable D2.3, Energy Aware Radio and neTwork tecHnologies (2010)

21. B Badic, T O'Farrrell, P Loskot, J He, Energy efficient radio access architectures for green radio: large versus small cell size deployment, in Vehicular Technology Conference Fall (VTC 2009-Fall), 2009 IEEE 70th, IEEE, pp. 1-5 (2010)

22. AD Wyner, Shannon-theoretic approach to a Gaussian cellular multipleaccess channel. Information Theory, IEEE Transactions on. 40(6), 1713-1727 (1994). doi:10.1109/18.340450

doi:10.1186/1687-1499-2012-193

Cite this article as: Wang et al:: Low energy indoor network: deployment optimisation. EURASIP Journal on Wireless Communications and Networking 2012 2012:193.

\section{Submit your manuscript to a SpringerOpen ${ }^{\mathcal{O}}$ journal and benefit from:}

- Convenient online submission

- Rigorous peer review

- Immediate publication on acceptance

- Open access: articles freely available online

- High visibility within the field

- Retaining the copyright to your article

Submit your next manuscript at $\gg$ springeropen.com 